\title{
Cervical canal morphology : effects of neck flexion in normal condition
}

- New elements for biomechanical simulations and surgical management -

Patrice Sudres (1,4), Morgane Evin (1,4), Pierre-Jean Arnoux $(1,4)$, Virginie Callot $(2,3,4)$

1. Laboratoire de Biomécanique Appliquée, UMRT24 AMU/IFSTTAR, Marseille, France;

2. Aix-Marseille Université, CNRS, CRMBM, Marseille, France;

3. APHM, Hôpital Universitaire Timone, CEMEREM, Marseille, France ;

4. iLab-Spine - Laboratoire International en Imagerie et Biomécanique du Rachis, Marseille, France \& Montréal, Canada.

Corresponding author : Morgane EVIN

\section{Address:}

Laboratoire de Biomécanique Appliquée

IFSTTAR/Université Aix-Marseille

UMR T24

Faculté de Médecine Nord

Boulevard Pierre DRAMART

13016 MARSEILLE CEDEX 20

Tel : (00 33) 491658006

Fax : (00 33) 491658019

E-Mail : morgane.evin@ifsttar.fr 
The Manuscript submitted does not contain information about medical device(s)/drug(s).

\section{Acknowledgments}

This work was supported by CARNOT STAR Institute, CNRS (Centre National de la Recherche Scientifique) and by Aix-Marseille University. This work was performed within a laboratory member of France Life Imaging network (grant ANR-11-INBS-0006). The authors would like to thank Lauriane Pini, Véronique Derderian, and Claire Costes for MR study logistics, as well as Amira Trabelsi and Yvan Petit for helpfull discussions. 


\section{Abstract (Max 300 words)}

Study Design. Continuous measurements and computation of absolute metrics of cervical subarachnoid space (CSS) and spinal cord (SC) geometries proposed are based on in vivo magnetic resonance imaging and 3D reconstruction.

Objective. The aim of the study is to offer a new methodology to continuously characterize and to quantify the detailed morphology of the CSS and the cervical SC in 3D for healthy subjects in both neutral supine and flexion.

Summary of Background Data. To the best of our knowledge, no study provide a morphological quantification by absolute indices based on the 3D reconstruction of SC and CSS thanks to in vivo magnetic resonance imaging. Moreover, no study provide a continuous description of the geometries.

Methods. Absolute indices of SC (cross-sectional area, compression ratio, position in the canal, length) and of CSS (cross-sectional area, occupational ratio, lengths) were computed by measures from 3D semi-automatic reconstructions of high resolution in vivo magnetic resonance images (3D T2-SPACE sequence) on healthy subjects ( $N=11)$ for two postures : supine neutral and flexion neck positions. The variability induced by the semi-automatic reconstruction and by the landmarks positioning were investigated by preliminary sensitivity analyses. Inter and intra-variability were also quantified on a randomly chosen part of our population $(\mathrm{N}=5)$.

Results. The length and cross-sectional area of SC are significantly different $(p<0.05)$ in flexion compared to neutral neck position. Spinal cord stays centered in the canal for both postures. However, the cross-sectional area of CSS is submitted to low variation after C3 vertebra for both postures. OR and $\mathrm{CR}$ after $\mathrm{C} 3$ are significantly lower in flexion.

Conclusions. This study presented interpretations of morphological measures :

1) left-right stability (described by the Left-Right eccentricity index) ensured by the denticulate ligaments and the nerve roots attached to the dural sheaths, 2) a Poisson effect of the SC was partially notified through its axial (AP diameter, OR, CR) and its longitudinal geometrical descriptions (LSC). Such 
morphological data can be useful for geometrical finite element modeling and could now be used to compare with injured or symptomatic subjects. 
Key points. (3-5 full sentences of the main pts of the article)

- The mechanical roles of the structures inside the vertebral canal in flexion : left-right stability (described by the LR eccentricity index) ensured by the denticulate ligaments and the nerve roots attached to the dural sheaths.

- A Poisson effect of the SC was partially highlighted through its axial (AP diameter, OR, CR) and its longitudinal geometrical descriptions (LSC).

- Such morphological data can be useful for geometrical finite element modeling of the canal in terms of boundaries, initial conditions as well as for an optimization of the mechanical properties by geometrical calibration of the model. 
Précis. (Submit a short description of the manuscript to appear in the Table of Contents, 3 sentences Max 50 words. )

The aim of the study is to offer a new methodology quantifying continuously the detailed morphologies of the cervical subarachnoid space and the spinal cord for healthy subjects in both neutral supine and flexion. In vivo magnetic resonance imaging and 3D semi-automatic reconstruction provide through computation of absolute metrics for both geometries. 


\section{1. Introduction}

Magnetic Resonance Imaging (MRI) has become a gold standard method to understand different physiological mechanisms of the spine. In the context of degenerative process, Kinematic MRI (kMRI), or postural MRI, which refers to imaging of the spine at different neck positions, is largely used to diagnosis positiondependent alterations that could not be detected in normal posture. It is mostly applied qualitatively to visualize cervical canal narrowing or spinal impingement by degenerated disk in flexion for instance, however little is known concerning the dynamical changes occurring at the level of the healthy cervical subarachnoid space(CSS) and spinal cord (SC) tissues.

Considering the limited spatial resolution that could be reached on conventional MR system (1.5 or 3T), the subarachnoid space is often simplified as a dural tube or as a space in the sagittal plane between the spinal cord and the anterior and posterior parts of the canal. Using this assumption, Kato et al. provided a large data base of MRI morphological measurements and showed the influence of the age and the gender on the occupation rate of the spinal cord in the dural tube on a large population ${ }^{5}$. Dahlan et al. ${ }^{6}$ established normal values of cervical canal diameters for young military subjects. Others studies focused on the SC location in the cervical canal based on two-dimensional (2D) visualization analysis for heathy subjects ${ }^{7}$ and for symptomatic subjects ${ }^{8}$. CSS and SC characterization at specific spine levels (mid-vertebrae, discs) can also be found, with different parameters such as compression ratio, cross-sectional area and spinal cord displacement that have been used in clinical research context ${ }^{1,2,3,4}$. In patients with Cervical Spondylotic Myelopathy (CSM), area of the SC, as well as antero-posterior and left-right diameters were described ${ }^{13,14}$ so as to evaluate the cord compression in the canal. When characterizing changes in posture, some articles considered a quantification of the strain of cord while others considered displacement measurements. Indeed, longitudinal strain of human cervical cord can reach a magnitude of $5 \%$ and $20 \%$ for, respectively, flexion and extension ${ }^{9,10,11,12}$, respectively.

In this study, based on 3D MR acquisitions in healthy subjects in both neutral supine and flexion, we propose a new methodology to characterize and to quantify the morphology of the CSS and the cervical SC in 3D for healthy subjects in both neutral supine and flexion. This approach aims at providing accurate and detailed morphological data (cross-sectional area, eccentricity indices, and displacements of the cord and canal) in order to have a deeper understanding of the impact of the flexion in the cervical canal. 


\section{Method}

\section{MRI acquisition}

Eleven european healthy subjects ( 5 females and 6 males, mean age : $30+/-6 y$, BMI : $23.86+/-1.78$ $\mathrm{kg} / \mathrm{m}^{2}$ ) were scanned using a 3T MR system (Siemens Healthineers, Erlangen, France) equipped with head, neck and spine coils. Subjects were first examined with the neck in neutral and flexion positions, using a dedicated homemade device (device flexion up to $40^{\circ}$ ). Images were acquired using a sagittal 3D $\mathrm{T}_{2}$-SPACE sequence (Sampling Perfection with Application optimized Contrasts using different flip angle Evolution) with the following parameters: voxel size $\left(1 \times 1 \times 1 \mathrm{~mm}^{3}\right)$, field-of-view $\left(256 \times 256 \mathrm{~mm}^{2}\right)$, echo time (TE : 124 $\mathrm{ms})$, repetition time (TR : $1500 \mathrm{~ms}$, ), total acquisition time $(6 \mathrm{~min})$. The MR protocol was approved by the institutional ethics committee and all subjects signed the informed consents. The cohort was chosen excluding history, presence of cervical pathologies or deformations.

\section{Segmentation and anatomical markers}

The SC was first segmented in 3D by a semi-automatic approach using the Spinal Cord Toolbox (SCT, 3.1.1 $)^{15}$ and providing $\mathrm{SC}$ centerline. The outer canal border, assumed as the dura mater, was then segmented by a semi-automatic approach from ITK-SNAP software ${ }^{16}$. The 3D CSS was defined as the space between the 3D canal segmentation and the 3D SC segmentation [Fig. 1-a,b].

Eleven landmarks [Fig. 1-b] were selected, by one operator on the middle slice of the sagittal view, using 3D Slicer 4.8.17 for the delimitation of the segment of interest:

1) "Reference landmarks": at the top of the dens of C2 (axis) and at the posterior extremity of C7 inferior endplate;

2) "Cervical vertebral landmarks", at the posterior extremities of each endplate (superior and inferior, C2 to C6).

\section{Data preparation, indices and lengths computation}

The post-processing steps performed using in-house Matlab codes (R2018a version, Matlab ${ }^{\circledR}$ MathWorks,1984) are summarized in [Fig. 1] and were performed for both neutral and flexion positions.

Both 3D segmentations (dural tube and SC raw surface meshes) were smoothed using $(\lambda: 1, \sigma: 0)^{18}$. 
Morphological parameters were computed from anatomical markers and centerline coordinates in less than 10 s per subject (Dell Latitude 7480, Intel ${ }^{\circledR}$ Core $^{\mathrm{TM}}$ i7-7600U CPU - 2.80GHz/2.90GHz, RAM : 8Go). The boundaries of the SC and the canal were interpolated as the intersection ${ }^{19}$ of the surface meshes and meshed planes orthogonal to the centerline [Fig. 2-c] using 3D splines. Then, the CSA of the SC, canal and CSS (CSA $A_{S C}, C S A_{\text {canal }}, C S A_{C S S}$, respectively) were computed (see [1]).

The cord transverse and antero-posterior diameters $\left(\varnothing \mathrm{T}_{S C}, \emptyset \mathrm{AP}_{S C}\right.$ respectively), as well as distance between the SC and the canal in anterior $(A)$, posterior $(P)$, right $(R)$ and left $(L)$ directions were computed from the most eccentrical points in AP (4) and LR (5) directions. Compression (CR - 2) and occupation (OR 3) ratios, as well as eccentricity indices were then derived from these metrics ${ }^{7}$ (see [2] to [5]).

$$
C S A_{C S S}=C S A_{\text {canal }}-C S A_{S C}
$$

$$
L R_{\text {eccenticity }}=\left(\frac{L-R}{L+R}\right) * 100
$$

An $A P_{\text {eccenticity }}>0(<0)$ indicates that the $\mathrm{SC}$ is anteriorly (posteriorly) positioned.

Similarly, a $L R_{\text {eccenticity }}>0(<0)$ indicates that the SC is located on the right (left) side of the canal.

Three specific lengths were also computed in both positions ${ }^{20,21}$ : the length of the cervical spinal cord (LSC), length of the cervical spinal anterior column (LAC) and length of the cervical spinal posterior column (LPC). They were respectively delimited by the caudal side of the pons to C7 inferior endplate, the C2 dens process to $\mathrm{C7}$ inferior endplate, and the top of $\mathrm{C} 1$ posterior arch to $\mathrm{C7}$ inferior endplate. Three 3D splines passing through the points positioned manually by an operator to define each curvature were computed to get more accurate lengths.

\section{Inter-operator variability, intra-operator variability and statistical analyses process}

The comparison between $C S A_{\text {canal }}$ measurements derived from ITK SNAP and manual segmentation 
was provided in 5 subjects randomly selected [Tab. 1] to assess the accuracy of the semi-automatic segmentation. Additional information on the reliability of the SC segmentation and $C S A_{S C}$ can be found in literature ${ }^{15}$. Inter- (by 2 raters) and intra-operator (at 2 different time points -operator 1 ) variabilities of 3D

\section{Results}

\section{Segmentation and inter-operator reliability}

The maximum error value between manual and automatic canal CSA measurements was found for the C2 dens reaching $25.06 \%$ while for the others cervical levels, the canal CSA relative errors reminded lower than $18 \%$. The standard deviation was steady around 15\% [Tab. 5 - Supplemental Digital Content].

The maximal distance, when considering inter-operator differences, was found of $2.29 \mathrm{~mm}$ (corresponding to an error of two $1 \mathrm{~mm}$-isotropic voxels) in neutral position and $4.29 \mathrm{~mm}$ (ie. 4 voxels) in neutral position and $3.62 \mathrm{~mm}$ (ie. 4 voxels) in flexion.

Consequences of inter/intra-operator differences in landmarks positioning were additionally assessed on four metrics $\left(C S A_{S C}, C S A_{C S S}, A P_{\text {eccenticity }}\right.$ and $\left.L R_{\text {eccenticity }}\right)$. The first tenth of the cervical segment (C1C2 functional unit) presented high variability (gray zone [Tab. 1]), whereas mean differences were less than $5 \%$ of the measured values otherwise, except for the $C S A_{C S S}$ (inter-operator mean relative error of $7.5 \%$ ). 
Cross-sectional areas and occupational ratio

The mean $C S A_{C S S}$ evolution along the canal after normalization in abscissa is depicted [Fig. 2]. The abscissa represents the averaged distances of each "cervical vertebral landmarks" from the top "reference landmarks" for all the subjects normalized using :

$$
Y=\frac{X-\min (X)}{\max (X)-\min (X)},
$$

with $\mathbf{Y}$ : the normalized vector of the abscissa.

$\mathbf{X}$ : the vector of mean values of the distances on the centerline from the top projected "reference landmark" to the projected "cervical vertebral landmark".

CSA rapidly decreased from C2 dens to $\mathrm{C} 2$ inferior endplate (from $481.6 \mathrm{~mm}^{2}$ to $177.5 \mathrm{~mm}^{2}$ in neutral position ; $379.8 \mathrm{~mm}^{2}$ to $157.9 \mathrm{~mm}^{2}$ in flexion), with minimal values around $\mathrm{C} 3$, followed by a low increasing and oscillating phase to reach $157 \mathrm{~mm}^{2}$ in neutral and $164 \mathrm{~mm}^{2}$ in flexion. CSA differences between both postures were found significant $(p<0.05)$ for $\mathrm{C} 1-\mathrm{C} 2, \mathrm{C} 5, \mathrm{C6}, \mathrm{C7}$ described in (cf. [Tab. 4]).

The mean of $C S A_{S C}$ for both postures is given on [Fig. 5]. In flexion, it was systematically lower than in neutral position and differences were found significant $(p<0.05)$ for all local locations (see [Tab. 4]).

The mean $\mathrm{OR}$ increased from $\mathrm{C} 2$ dens to $\mathrm{C} 2$ inferior endplate for the both neutral and flexion postures (from 0.18 to 0.32 and 0.19 to 0.32 , respectively). After the superior $\mathrm{C} 3$ endplate $(0.33$ - neutral ; 0.35 flexion), the flexion OR decreases faster than in neutral position with an oscillating behavior until the $\mathrm{C} 7$ inferior vertebrate endplate $(0.31$ - neutral ; 0.22 - flexion). Large standard deviations could be noticed $( \pm 8$ $30 \%$ - neutral ; $\pm 13-45 \%$ - flexion) [Fig. 2]. OR differences between both postures were found significant $(p<0.05)$ for $\mathrm{C} 1-\mathrm{C} 2, \mathrm{C} 5, \mathrm{C} 6, \mathrm{C7}$ described in (cf. [Tab. 4 - Supplemental Digital Content]). Eccentricity indices and compression ratio

The mean AP and LR eccentricity indices, as well as the CR index along the normalized length of the canal are depicted [Fig. 3]. The first tenth of the presented curves ( 0 to 0.1 in abscissa) will not be considered in 
further analysis because of the difficulty of the post-processing algorithm (section 2.3) to be efficient with

the small complex geometry of the subarachnoid space (cisterna magna/pontis/interpeduncularis, medulla oblongata) between the foramen magnum and the $\mathrm{C} 2$ dens.

Between the $\mathrm{C} 2$ dens and the inferior $\mathrm{C} 2$ endplate, the $A P_{\text {eccenticity }}$ index decreased steadily with the same behavior for both neutral and flexion postures (from $52 \%$ to $-22 \%$ and $58 \%$ to $-24 \%$, respectively). After the C3 superior endplate, mean $A P_{\text {eccenticity }}$ index in neutral was systematically lower than in flexion position. [Fig. 3-Top]. Differences between both postures were found significant $(p<0.05)$ for $\mathrm{C} 1-\mathrm{C} 2, \mathrm{C} 5, \mathrm{C} 6$, C7 described in (cf. [Tab. 4]).

The $L R_{\text {eccenticity }}$ index constantly oscillated around $0 \%$ and $8 \%$ respectively in neutral and flexion neck postures respectively [Fig.3-Middle]. No significant difference in $L R_{\text {eccenticity }}$ index between the postures was found $(0.08<p<0.97)$ except for the vertebral level C1-C2 (cf. [Tab. 04]).

The $\mathrm{CR}$ index decreased steadily between $\mathrm{C} 2$ dens and to $\mathrm{C} 7$ inferior endplate for both posture. The $\mathrm{CR}$ for the flexion case was systematically lower than in neutral position, except along the C3 vertebra [Fig. 3Bottom]. CR differences between neutral and flexion neck postures were significant $(p<0.05)$ locally for the vertebral levels $\mathrm{C4}, \mathrm{C5}, \mathrm{C6}$ and for the mid-levels of intervertebral discs values of $\mathrm{C4} / \mathrm{C} 5$ to $\mathrm{C6} / \mathrm{C7}$ [Tab. 04]. Anterior/Posterior columns and of the spinal cord

The mean length values were measured equal to $118 \pm 5 \mathrm{~mm}$ and $120.3 \pm 7.6 \mathrm{~mm}$ for LAC, $107 \pm 6$ and 120.4 $\pm 6.4 \mathrm{~mm}$ for LPC, $142 \pm 7 \mathrm{~mm}$ and $159.4 \pm 11.5 \mathrm{~mm}$ for LSC in neutral and flexion neck postures, respectively, with significant differences between both the postures in each case $(p<0.05)[$ Fig. 4, Tab. 4].

\section{Discussion}

This study proposes a new approach to collect detailed anatomical measurements of the cervical canal. Using MRI segmented 3D geometrical reconstructions, it was possible to investigate the morphological changes between neutral supine and neck-flexion positions. Different geometrical metrics (CSA, eccentricity indices, ratios) related to the SC and the CSS could be derived. The most noticeable results are summarized in [Tab. 3].

\section{Cord and CSS kinematical description}

Firstly, the low oscillating behavior of $C S A_{C S S}$ and OR indices along the spinal segment in [Fig. 2,3] describes the geometry of dura mater at each spine level : nerve root funnel-shaped lateral extensions of 
sheaths leave the dura mater as spinal ganglions toward the peripheral nervous system ${ }^{22}$, hence including

pseudo-periodic fluctuations in the CSS outer border.

Secondly, the kinematic of the cord is not only induced by the flexion itself but also by the surrounding structures. The spinal cord being fixed to the superior anatomical structures (pons, medulla) on one hand and to the filum terminale on the other hand, it is subject to tensile forces applied in both cranio-caudal and antero-posterior directions when flexed. Previous studies reported up to 2-mm displacements of the medulla oblongata and the pons in flexion ${ }^{23}$. Here a "stretching" phenomenon $(+13 \%$ for LSC in flexion with respect to neutral position, after $\mathrm{C} 2$ endplate) occurs while the $\mathrm{SC}$ is pull through the anterior side of the spine column, as reflected by the $A P_{\text {eccenticity }}$ and $L R_{\text {eccenticity }}$ [Fig 3-a,b]. The nerve roots attached in the dural sheaths could be limiting the longitudinal motion and allow an elongation that follows the spinal motion. The SC lateral position stays globally centered (slight shift on the left : 2-3\% from the center of the canal), in line with the literature ${ }^{7}$, and similar in neutral and in flexion. Indeed, both denticulate ligaments ${ }^{24,}$ 25 between the arachnoid (assumed to be stick to the dura mater) and the pia mater, and nerve roots leaving the SC dorsal and ventral side by pairs and exiting the CSS by the dural sheath, insure coronal stabilization of the SC.

Thirdly, the high reduction of $C S A_{C S S}$ from $\mathrm{C2}$ dens to $\mathrm{C} 3$ vertebra in flexion position compared to the neutral one appears to be induced by the high mobility of the upper cervical segment ${ }^{26}$. After the C3 vertebra, the $C S A_{S C}$ was globally steady while the $C S A_{C S S}$ decreased. In addition, the action of the yellow and posterior longitudinal ligaments inside the vertebral should be noticed ${ }^{27}$ while explaining the CSS modification ${ }^{28}$.

\section{Toward cord and spine biomechanical description}

The geometrical evolution described by the CR index was intrinsically linked to the mechanical properties of the spinal cord. Indeed, the decrease of the CR from C4 to C6 observed in flexion depicts a significant strain of the SC from $3 \%$ to $7 \%$. Such results underline the "stretching" phenomenon previously mentioned. This is further confirmed by the $13 \%$ increase of the LSC in flexion, in line with literature ${ }^{10,29,21,9}$. The "stretching" phenomenon results from the the Poisson effect described as the amount of lateral contraction against the amount of axial elongation experienced by a material under the action of a force. The three OR, CP, and LSC metrics could overcome the current lack of mechanical characterization and could be used for biomechanical modeling validation. Such Poisson effect can induce some local stresses or 
damages in the structure near the zone where the cord is attached, namely nerve roots and denticulate

ligaments ${ }^{9}$.

The LAC and LPC values (respectively $+2 \%$ and $+12 \%$ in flexion) are also in line with literature ${ }^{20}$ (respectively $5 \%$ and $8 \%$ ). The LSC metric in comparison to LAC and LPC ones shows that the SC follows the motion of the canal in freeing more space posteriorly in flexion $\left(-22 \%<A P_{\text {eccenticity }}<47 \%\right)$ than in neutral position $\left(-22 \%<A P_{\text {eccenticity }}<7 \%\right)$ after $\mathrm{C} 2$ vertebra. Validation and further use of spine models, especially with finite element modeling, require advanced knowledge of spinal cord interaction with surrounding structures. Hence the data related to the location of the cord and its space distribution obtained in this study could provide a strong added value for model design. In addition, spinal cord positioning and length variations are also providing additional indirect information (based on Poisson ratio effects) to support further modeling choices. Altogether, our study provide the necessary measurements [Tab. 4] in a healthy adult population in physiologically conditions for such validation.

\section{Clinical aspects}

In this study, we presented ten morphological parameters were used to describe the postural modifications occurring in the canal. These parameters were chosen in relation to their use in a clinical research context. Indeed, transverse area criteria corresponding to our $\operatorname{CSA}_{S C}, \operatorname{CSA}_{C S S}, \operatorname{CSA}_{\text {Canal }}$, $\emptyset \mathrm{T}_{S C}, \emptyset \mathrm{AP} S$ and CR, are used for CSM description ${ }^{13,14}$. CSM, disc herniation, osteophytes (bony outgrowth) can also cause reduction of the canal and an occupation ratio (sagittal diameters ratio of spinal cord over canal, or ratio of spinal cord over canal area corresponding to our OR index) could be useful to quantify the degree of compression ${ }^{30,31}$. Moreover, additional parameters related to the location of the spinal cord in the canal ( $A P_{\text {eccenticity }}$ and $L R_{\text {eccenticity }}$ indices) and the characteristic lengths (LSC, LAC, LPC) complete this physiological database of healthy subjects that could now be used to compare with injured or symptomatic subjects. This methodology and database open the perspective of more accurate clinical indices. Limitations

The first limitation of our methodology is that the MR acquisitions were performed in supine position, which is not a daily physiological posture. A previous study showed no significant difference of the spinal cord length between erect and supine position ${ }^{29}$. However, with cervical lordosis changes, a slight difference between erect and supine position is expected for SC position in the CSS and eccentricity indices. 
Nonetheless, as the cerebrospinal fluid mechanical properties are similar to water properties ${ }^{32,33}$

\title{
5. Conclusion
}

\author{
A new methodology allowing a detailed description of the vertebral canal morphology thanks to high \\ resolution 3T-kMRI was developed. It allowed characterizing the CSS dynamic behavior through 10 \\ geometrical metrics. Reference values in healthy population are provided in [Tab. 4]. \\ The mechanical roles of the different structures inside the vertebral canal in flexion were also confirmed
} through geometric measurements changes : 1) left-right stability (described by the LR eccentricity index) ensured by the denticulate ligaments and the nerve roots attached to the dural sheaths, 2) a Poisson effect of the SC was partially notified through its axial (AP diameter, OR, CR) and its longitudinal geometrical description (LSC). Such morphological data can be useful for geometrical finite element modeling of the canal in terms of boundaries or initial conditions or for an optimization of the mechanical properties by geometrical sensitivity analysis. Confronting this methodology and these results in a further study with a pathological cohort (CSM, post-traumatic population) is crucial to refine these clinical criteria in the medicosurgical management. 


\section{References}

1. Kato F, Yukawa Y, Suda K, et al. Normal morphology, age-related changes and abnormal findings of the cervical spine. Part II: Magnetic resonance imaging of over 1,200 asymptomatic subjects. Eur Spine J. 2012;21(8):1499-1507.

2. Dahlan RH, Saputra PAE, Yudoyono FF, et al. Morphometric Analysis of the Corpus, Spinal Canal and Torg Ratio Using Midsagittal Cervical Vertebrae Computed Tomography Scan: Indonesian Population. International Journal of Integrated Health Sciences. 2014;2(1):36-39.

3. Fradet L, Arnoux P-J, Ranjeva J-P, et al. Morphometrics of the entire human spinal cord and spinal canal measured from in vivo high-resolution anatomical magnetic resonance imaging. Spine. 2014;39(4):E262-269.

4. Kar M, Bhaumik D, Ishore K, et al. MRI Study on Spinal Canal Morphometry: An Indian Study. J Clin Diagn Res. 2017;11(5):AC08-AC11.

5. Wolf K, Hupp M, Friedl S, et al. In cervical spondylotic myelopathy spinal cord motion is focally increased at the level of stenosis: a controlled cross-sectional study. Spinal Cord. 2018;56(8):769776.

6. Kong L, Bai J, Zhang B, et al. Predictive factors of symptomatic lumbar canal stenosis in patients after surgery for cervical spondylotic myelopathy. Therapeutics and Clinical Risk Management.

7. Tykocki T, du Plessis J, Wynne-Jones G. Correlation between the severity of myelopathy and cervical morphometric parameters on dynamic magnetic resonance imaging. Acta Neurochir. 2018;160(6):1251-1258.

8. Tykocki T, du Plessis J, Wynne-Jones G. Analysis of Morphometric Parameters in Cervical Canal Stenosis on Neutral and Dynamic Magnetic Resonance Imaging. World Neurosurgery. 2018;114:e317-e322.

9. Hu P, He Z, Cui J, et al. Pathological changes of cervical spinal canal in cervical spondylotic myelopathy: A retrospective study on 39 cases. Clinical Neurology and Neurosurgery. 2019;181:133-137. 
10. Smith J, Lafage V, Ryan D, et al. Association of Myelopathy Scores With Cervical Sagittal Balance and Normalized Spinal Cord Volume: Analysis of 56 Preoperative Cases From the AOSpine North America Myelopathy Study. Spine. 2013;38(22S).

11. Stoner KE, Abode-lyamah KO, Magnotta VA, et al. Measurement of in vivo spinal cord displacement and strain fields of healthy and myelopathic cervical spinal cord. Journal of Neurosurgery: Spine. 2019;31(1):53-59.

12. Yuan Q, Dougherty L, Margulies SS. In vivo human cervical spinal cord deformation and displacement in flexion. SPINE. 1998;23(15):1677-1683.

13. Bilston LE. The biomechanics of the spinal cord during traumatic spinal cord injury. 1994. https://core.ac.uk/display/76367966.

14. Bilston LE, Thibault LE. Biomechanics of cervical spinal cord injury in flexion and extension: A physical model to estimate spinal cord deformations. International Journal of Crashworthiness. $1997 ; 2(2): 207-218$.

15. De Leener B, Lévy S, Dupont SM, et al. SCT: Spinal Cord Toolbox, an open-source software for processing spinal cord MRI data. Neurolmage. 2017;145:24-43.

16. Yushkevich PA, Piven J, Hazlett HC, et al. User-guided 3D active contour segmentation of anatomical structures: Significantly improved efficiency and reliability. Neurolmage. 2006;31(3):1116-1128.

17. Kikinis R, Pieper SD, Vosburgh KG. 3D Slicer: A Platform for Subject-Specific Image Analysis, Visualization, and Clinical Support. In: Jolesz FA, ed. Intraoperative Imaging and Image-Guided Therapy. New York, NY: Springer New York; 2014:277-289.

18. Desbrun M, Meyer M, Schröder P, Barr AH. Implicit Fairing of Irregular Meshes Using Diffusion and Curvature Flow. In: Proceedings of the 26th Annual Conference on Computer Graphics and Interactive Techniques. SIGGRAPH '99. New York, NY, USA: ACM Press/Addison-Wesley Publishing Co.; 1999:317-324. 
19. Tropp O, Tal A, Shimshoni I. A fast triangle to triangle intersection test for collision detection. Journal of Visualization and Computer Animation. 2006;17:527-535.

20. Jha SC, Miyazaki M, Tsumura H. Kinetic change of spinal cord compression on flexion-extension magnetic resonance imaging in cervical spine. Clinical Neurology and Neurosurgery. 2018;174:8691.

21. Endo K, Suzuki H, Nishimura H, et al. Kinematic Analysis of the Cervical Cord and Cervical Canal by Dynamic Neck Motion. Asian Spine J. 2014;8(6):747-752.

22. Frykholm R. Deformities of Dural Pouches and Strictures of Dural Sheaths in the Cervical Region Producing Nerve-Root Compression: A Contribution to the Etiology and Operative Treatment of Brachial Neuralgia. Journal of Neurosurgery. 1947;4(5):403-413.

23. Ji S, Margulies SS. In vivo pons motion within the skull. Journal of Biomechanics. 2007;40(1):92 99.

24. Ceylan D, Tatarlı N, Abdullaev T, et al. The denticulate ligament: anatomical properties, functional and clinical significance. Acta Neurochir. 2012;154(7):1229-1234.

25. Polak-Kraśna K, Robak-Nawrocka S, Szotek S, et al. The denticulate ligament - Tensile characterisation and finite element micro-scale model of the structure stabilising spinal cord. Journal of the Mechanical Behavior of Biomedical Materials. 2019;91:10-17.

26. Nightingale RW, Winkelstein BA, Knaub KE, et al. Comparative strengths and structural properties of the upper and lower cervical spine in flexion and extension. Journal of Biomechanics. 2002;35(6):725-732.

27. Johnson RM, Crelin ES, White AA, et al. Some new observations on the functional anatomy of the lower cervical spine. Clin Orthop Relat Res. 1975;(111):192-200.

28. Holmes A, Han ZH, Dang GT, et al. Changes in Cervical Canal Spinal Volume During In Vitro FlexionExtension. Spine. 1996;21(11):1313. 
29. Kuwazawa Y, Pope MH, Bashir W, et al. The Length of the Cervical Cord: Effects of Postural Changes in Healthy Volunteers Using Positional Magnetic Resonance Imaging. Spine. 2006;31(17):E579.

30. Sun K, Wang S, Sun J, et al. Analysis of the Correlation Between Cerebrospinal Fluid Space and Outcomes of Anterior Controllable Antedisplacement and Fusion for Cervical Myelopathy Due to Ossification of the Posterior Longitudinal Ligament. World Neurosurgery. 2019;122:e358-e366.

31. Pratali RR, Smith JS, Ancheschi BC, et al. A Technique for Dynamic Cervical Magnetic Resonance Imaging Applied to Cervical Spondylotic Myelopathy: A Reliability Study. Spine. 2019;44(1):E26.

32. Bloomfield IG, Johnston IH, Bilston LE. Effects of Proteins, Blood Cells and Glucose on the Viscosity of Cerebrospinal Fluid. PNE. 1998;28(5):246-251.

33. Schiffer E, van Gessel E, Gamulin Z. Influence of sex on cerebrospinal fluid density in adults. British Journal of Anaesthesia. 1999;83(6):943-944.

34. Cadotte DW, Cadotte A, Cohen-Adad J, et al. Characterizing the location of spinal and vertebral levels in the human cervical spinal cord. AJNR Am J Neuroradiol. 2015;36(4):803-810. 
Abbreviations.

AP : antero-posterior

AVD VERT : spatial averaged value (over the whole vertebra)

$\mathrm{CP}$ : compression ratio

CSA : cross-sectional area

CSM : cervical spondylotic myelopathy

CSS : cervical subarachnoïd space

LAC : length of anterior column

LPC : length of posterior column

LR : left-right

LSC : length of spinal cord

MID-IVD : spatial value for cross-sectional plane located at the middle of the intervertebral disc

MID-VERT : spatial value for a cross-sectional plane mid-vertebra

MRI : magnetic resonance imaging

kMRI : kinematic magnetic resonance imaging

OR : occupational ratio

SC : spinal cord 


\begin{tabular}{|l|l|l|l|l|}
\hline $\begin{array}{l}\text { Intra-operator } \\
\text { differences }\end{array}$ & $\begin{array}{l}\text { CSA SC } \\
\text { [mm2] }\end{array}$ & $\begin{array}{l}\text { CSA CSS } \\
{[\mathbf{m m} 2]}\end{array}$ & $\begin{array}{l}\text { AP Eccentricity } \\
{[\%]}\end{array}$ & $\begin{array}{l}\text { LR Eccentricity } \\
{[\%]}\end{array}$ \\
\hline Neutral & $1.36 \pm 0.85 ;$ & $16.42 \pm 7.32 ;$ & $3.13 \pm 2.02 ;$ & $3.85 \pm 4.66 ;$ \\
& $0 / 2.69$ & $0.01 / 29.98$ & $0 / 6.56$ & $0.01 / 18.18$ \\
\hline & $2.36 \pm 0.97 ;$ & $2.26 \pm 2.17 ;$ & $2.11 \pm 2.15 ;$ & $2.15 \pm 2.03 ;$ \\
& $0.5 / 4.57$ & $0.02 / 11.27$ & $0.01 / 10.08$ & $0.02 / 11.58$ \\
\hline Flexion & $3.49 \pm 1.79 ;$ & $32.28 \pm 22.38 ;$ & $6.62 \pm 3 ;$ & $3.58 \pm 2.42 ;$ \\
& $0 / 6.92$ & $0.06 / 72.69$ & $0.05 / 11.79$ & $0.12 / 8.98$ \\
\hline & $1.1 \pm 1.26 ;$ & $4.81 \pm 4.56 ;$ & $3.54 \pm 3.11 ;$ & $2.93 \pm 2.78 ;$ \\
& $0.01 / 11.35$ & $0.03 / 24.68$ & $0.03 / 15.13$ & $0.01 / 14.53$ \\
\hline
\end{tabular}

Table 1

\begin{tabular}{|c|c|c|c|c|}
\hline & \multicolumn{2}{|c|}{ Inter-operator } & \multicolumn{2}{|c|}{ Intra-operator } \\
\hline & Neutral & Flexion & Neutral & Flexion \\
\hline $\begin{array}{l}\text { C1 odontoid } \\
\text { (ref pt) }\end{array}$ & $1.72 ; 0.52 / 4.26$ & $4.29 ; 1.47 / 6.19$ & $2.12 ; 0.56 / 4.02$ & $3.62 ; 2.36 / 6.22$ \\
\hline C2 inf & $2.03 ; 0.31 / 3.51$ & $6.4 ; 1.27 / 29.75$ & $1.44 ; 0.36 / 3.2$ & $2.64 ; 1.13 / 6.09$ \\
\hline C3 sup & $2.29 ; 1.13 / 3.82$ & $2.37 ; 0.37 / 3.37$ & $1.53 ; 0.87 / 3.83$ & $2.51 ; 0.86 / 5.67$ \\
\hline C3 inf & $2.05 ; 0.98 / 4.07$ & $2.61 ; 1.33 / 4.87$ & $2.02 ; 1 / 3.61$ & $2.49 ; 1.01 / 5.58$ \\
\hline C4 sup & $1.62 ; 0.27 / 3.01$ & $2.77 ; 1.77 / 3.4$ & $1.29 ; 0.42 / 2.37$ & $2.65 ; 1.36 / 5.07$ \\
\hline C4 inf & $2.03 ; 0.36 / 3.73$ & $2.61 ; 1.3 / 3.51$ & $1.63 ; 0.32 / 2.53$ & $2.6 ; 1.12 / 5.08$ \\
\hline C5 sup & $1.96 ; 0.74 / 3.52$ & $3.69 ; 1.32 / 12.06$ & $1.43 ; 0.72 / 2.77$ & $2.43 ; 1.2 / 5.01$ \\
\hline C5 inf & $2.12 ; 0.89 / 4.25$ & $2.99 ; 1.26 / 5.35$ & $1.29 ; 0.08 / 2.57$ & $2.85 ; 1.53 / 6.31$ \\
\hline C6 sup & $1.69 ; 0.9 / 2.73$ & $3.84 ; 1.54 / 14.01$ & $1.65 ; 1.03 / 2.4$ & $2.44 ; 0.73 / 5.02$ \\
\hline C6 inf & $1.61 ; 0.4 / 3.43$ & $2.84 ; 1.27 / 5.13$ & $1.31 ; 0.29 / 2.5$ & $2.49 ; 0.17 / 6.75$ \\
\hline C7 sup & $1.32 ; 0.38 / 2.39$ & $3.76 ; 1.3 / 14.49$ & $1.42 ; 0.5 / 2.62$ & $2.19 ; 0.67 / 5.05$ \\
\hline C7 inf (ref pt) & $1.32 ; 0.11 / 2.72$ & $2.51 ; 1.01 / 4.81$ & $1.47 ; 0.71 / 2.28$ & $2.43 ; 1.43 / 5.44$ \\
\hline
\end{tabular}

Table 2 


\begin{tabular}{|c|l|}
\hline Morphological parameters & \multicolumn{1}{|c|}{ Main position-dependent evolution } \\
\hline CSS CSA & $\begin{array}{l}\text { Significant decrease from C1 to C3 superior } \\
\text { endplate. From C3 to C7, low variation and } \\
\text { oscillating phase around } 157 \mathrm{~mm}^{2} \text { in neutral and } \\
162 \mathrm{~mm}^{2} \text { in flexion positions. }\end{array}$ \\
\hline SC CSA & $\begin{array}{l}\text { Significantly lower in flexion than in neutral supine } \\
\text { position. }\end{array}$ \\
\hline OR & $\begin{array}{l}\text { After the superior C3 endplate (0.33-neutral; } 0.35 \\
\text { - flexion), the flexion OR decreases faster than in } \\
\text { neutral position. }\end{array}$ \\
\hline CR & $\begin{array}{l}\text { The CR index decreased steadily between C2 dens } \\
\text { and to C7 inferior endplate for both posture. The } \\
\text { CR for the flexion case is systematically lower. }\end{array}$ \\
\hline AP eccentricity index & $\begin{array}{l}\text { Same location for the both postures before C3 } \\
\text { vertebra. After C3, in flexion the SC is more } \\
\text { anteriorly positioned with respect to the center of } \\
\text { the canal. }\end{array}$ \\
\hline LR eccentricity index & $\begin{array}{l}\text { Spinal cord centered (shift of 3\% from the center of } \\
\text { the canal) in the canal for the both postures. }\end{array}$ \\
\hline Characteristics lengths & $\begin{array}{l}\text { Significantly different in neck flexion than in neutral } \\
\text { supine position (+2 mm, 13mm and 18mm for the } \\
\text { anterior column (LAC), posterior column (LPC) and } \\
\text { spinal cord, respectively). }\end{array}$ \\
\hline
\end{tabular}

Table 3 


\section{Legends}

2

3

4

5

6

7

8

9

10

Table 1 : inter-and intra- mean relative errors for spinal cord (SC) and Cervical Subarachnoid Space (CSS) CSA and Anteroposterior (AP) and Left - Right (LR) eccentricity indices. The averaged value, standard deviation, minimum and maximum averaged value on the 5 subjects randomly selected are described as: [Mean \pm STD; Min/Max]. The grey zones represent the first tenth of the measures along the cervical cord and the white zones represent the rest.

Table 2 : Distance errors (L2-norm in $\mathrm{mm}$ ) per vertebra landmarks between operator 1 and the operator 2 (inter-operator variability), as well as between 2-measurements sessions by operator 1(intra-operator variability). For each cell, the results are presented as follow : [Mean ; Min/Max].

Table 3 : summary table of the main results for each index.

Supplemental Digital Content Table 4 : statistical summary [Mean \pm std ( $\mathrm{min} /$ max)] of each measured parameter (SC and CSS $C S A, O R, A P / L R$ eccentricity and $C R$ ) for both position (neutral in white, flexion in gray). For each one, three sub-values are given, corresponding to a spatial vertebral average of measures (AVD VERT, with C1 and C2 vertebrae merged: the C2 dens passing through the ring of $C 1$ vertebra), value picked up at the middle of the vertebra in the middle sagittal plan (MID VERT) and value picked up at the middle of the intervertebral disc in the middle sagittal plan (MID IVD), considering the disc below the mentioned vertebral level). The LAC, LPC and LSC parameters correspond to the lengths of the anterior canal, posterior canal and the spinal cord, along the whole cervical segment, respectively. *indicates $p<0.05$ (Paired samples Wilcoxon test).

Supplemental Digital Content Table 5 : table of CSA relative error [\%] between manual and automatic CSA measurements for 5 subjects randomly selected. Mean and standard deviation were computed by averaging all subjects for seven levels along the cervical spine.

Figure 1 - a) Sagittal view with superimposed 3D reconstruction of the SC (gold color) and the CSS (blue color) in flexion (left), and neutral (right) neck positions. The red dotted lines indicates the delimitation of the cervical segment (C1-C7) considered in this study. b) Main postprocessing steps, 3D reconstruction and 3D landmarks (left), orthogonal planes to the centerline all along the cervical segment (middle), boundaries points (zoom x5) extracted from the intersection between 3D reconstruction and the plane, with distance representations of eccentricity indices $(A, P, L, R, \varnothing A P, \varnothing T)$ (right). c) As in Jha et al. ${ }^{20}$, main steps to compute the length of the cervical spinal cord (LSC) attached to the caudal side of the pons in red, length of the cervical spinal anterior column (LAC) in yellow and length of the cervical spinal posterior column (LPC) in green (top : manual inputs, bottom : 3D-spline interpolation. 
33 Figure 2 - (Top) Mean Cross-Sectional Area $\left(\mathrm{CSA}, \mathrm{mm}^{2}\right.$ ) along the normalized cervical spinal normalized (see section 3.2 formula 6 for normalization process) segment of interest length, (bottom) Mean Occupation Ratio (\% defined as SC CSA over total canal CSA (SC CSA + CSS CSA)). For each subplot figure, the mean value of the neutral (blue continuous curve) and flexion (red continuous curve) cases are indicated with their standard deviations (dot curve). Mean normalized positions of the vertebral endplates (computed as the mean position of each vertebral endplates between all the subjects) are indicated with color boxes and as vertical lines (small dots in flexion and bigger dots in neutral).

Figure 3 - AP (top) and LR (middle) eccentricity indices along the normalized cervical cord, together with the Compression Ratio index (bottom). For each subplot figure, the mean value of the neutral (blue continuous curve) and flexion (red continuous curve) cases are indicated with their standard deviations (dotted curves). Mean normalized positions of the vertebral endplates are indicated as well, standard deviations are not indicated on the figure for a sake of clarity.

Figure 4 - Boxplots of characteristics lengths in the cervical segment, a) Length of the Anterior Column (LAC), b) Length of the Posterior Column (LPC) c) Length of the Spinal Cord (LSC). On each box, the central mark indicates the median (red line), and the bottom and top edges of the box indicate the 25th and 75th percentiles (horizontal blue lines), respectively. The whiskers extend to the most extreme data points not considered outliers, and the outliers are plotted individually using the red ' + ' symbol $\left({ }^{*} p<0.05\right)$.

Supplemental Digital Content Figure 5 - Spinal cord cross-sectional area (SC CSA) mean along the cervical. The mean value of the neutral (blue continuous curve) and flexion (red continuous curve) cases are indicated with their standard deviations (dotted curves). Mean normalized positions of the vertebral endplates are indicated as well standard deviations are not indicated on the figure for a sake of clarity. 


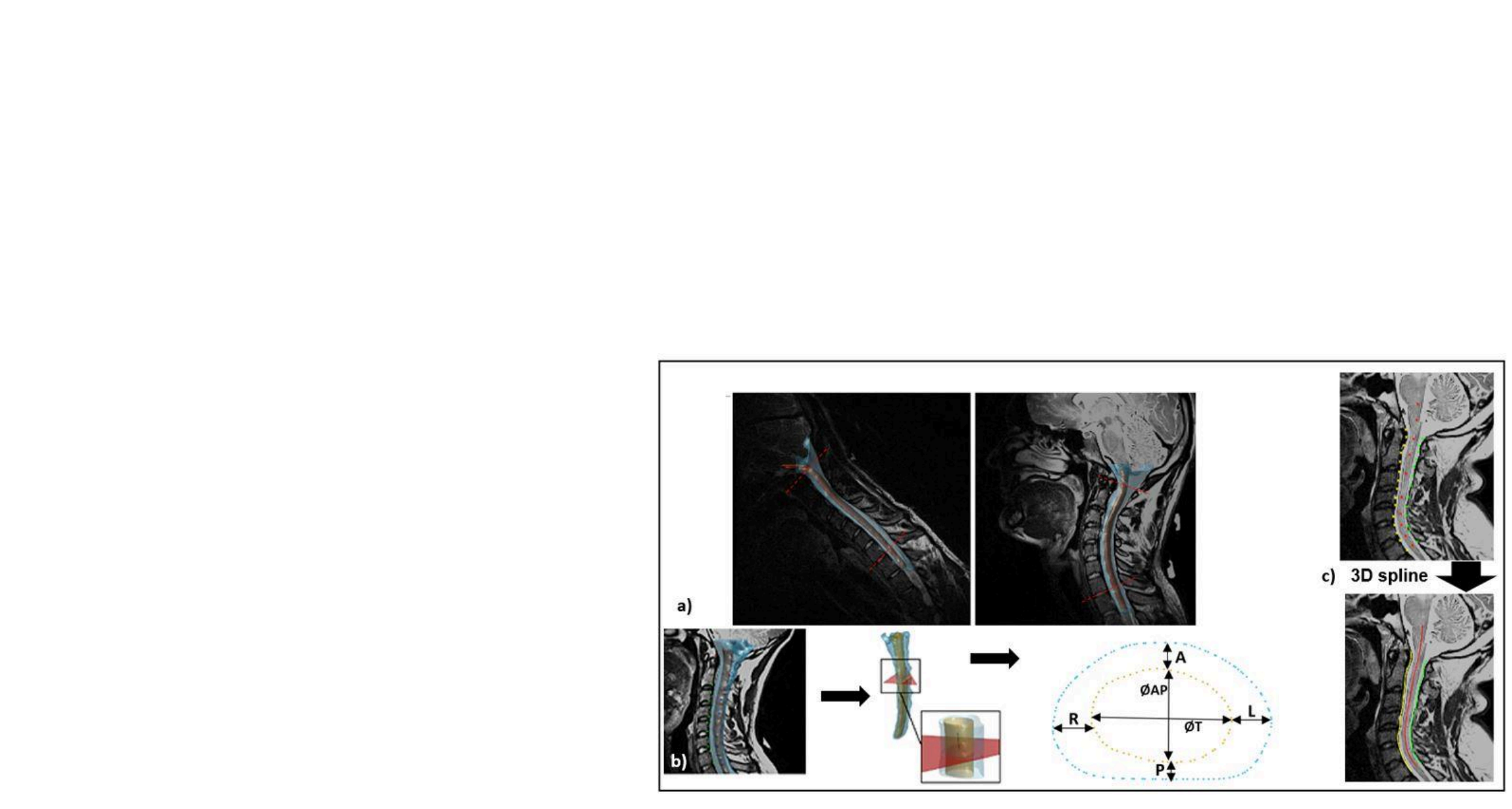




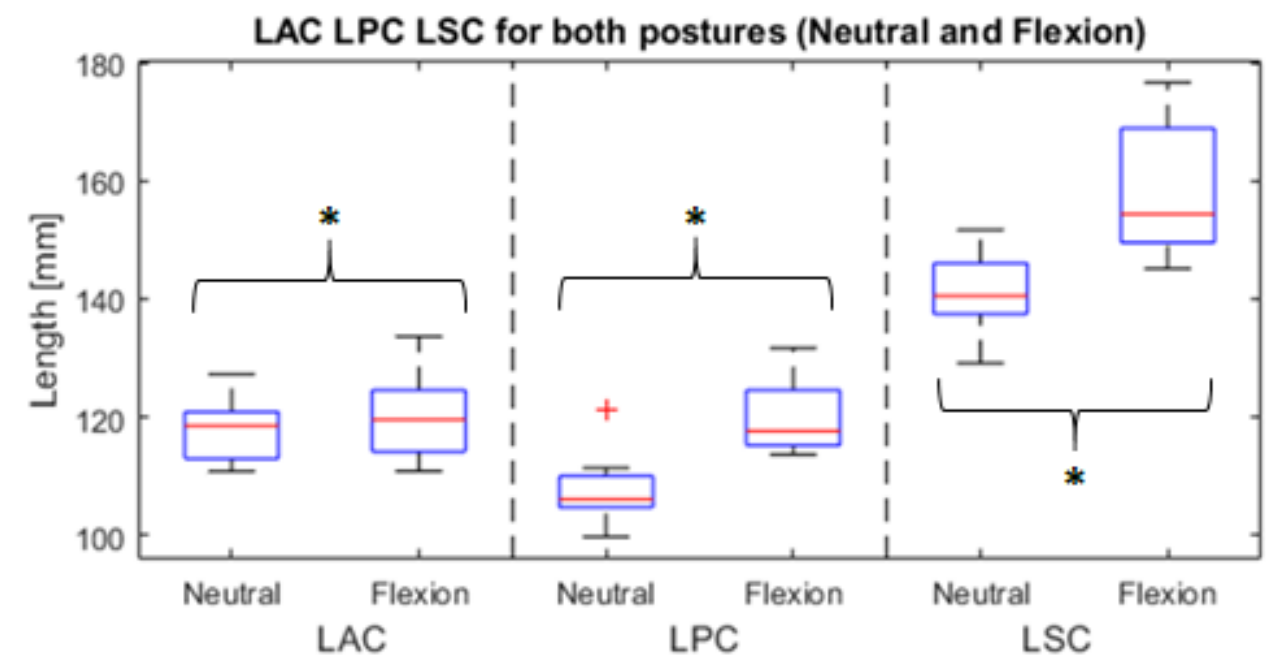

